\title{
Power Analog to Digital Converter for Voltage Scaling Applications
}

\author{
M.C.Gonzalez, M.Vasić, P.Alou, O.Garcia, \\ J.A. Oliver and J.A.Cobos \\ Centro de Electrónica Industrial \\ Universidad Politécnica de Madrid \\ Madrid, España \\ carmen.gsanchez@upm.es
}

\author{
H.Visairo \\ Systems Research Center, Mexico \\ Intel Corporation \\ Gnadalajara, México \\ horacio. visairo-cruz(â) intel.com
}

\begin{abstract}
In order to optimize energy efficiency, some applications require adapting supply voltage according to the work load requirements. For example, in high performance digital systems and in $R F$ systems, voltage scaling and modulation techniques have been adopted in order to achieve a more efficient processing of the energy. These techniques are based in rapidly adjusting the system supply voltage level. In order to achieve this, a topology which is capoble of achieving very fast changes of the output voltage is needed In this paper a PWM multiphase topology whose phases are coupled by using transformers is proposed to be used in an envelope elimination and restoration (EER) technique. The proposed topology can achieve very fast changes between discrete voltage steps so it can be cousidered as a power analog to digital converter.
\end{abstract}

\section{INTRODUCTION}

More efficient processing of the energy has been a matter of interest in many fields of power electronics since energy savings reflect directly in cost savings and higher autonony of mobile devices. For instance, commnnication and digital systems are two applications where a more efficient processing of the energy means higher autonomy of mobile devices. Techniqnes such as DVS (Dynamic Voltage Scaling) are employed in order to reduce the power consumption of high perfonnance digital systems; while in RF systems, EER (Envelope Elimination and Restoration) and envelope tracking techniques are employed. In these techniques, the power supply plays an important role, since the energy savings rely partly on the power supply ability to rapidly adjnst the outpnt voltage. Apart from adeqnately adjnsting the outpnt voltage, power supplies must accomplish other requirements in order to enable voltage modulation. Some of these requirements are the following ([1], [4]):

- Small size

- Fast dynamic response

- High efficiency over a wide load range

- Fast voltage transitions
- While in DVS it is also important for the power supply to exhibit very goxd static regulation, power supplies for RF systems should not interfere with the output spectrum of the transmitter.

A simplified block diagram of a digital system with DVS architecture and an RF system with envelope elimination and restoration (EER) technique are shown in Figure 1. In this paper, a PWM mnltiphase converter is proposed as a part of a power supply in a RF system. As stated, the reason to implement voltage modulation in RF systems is to improve the efficiency of the processing of the energy. In order to do this, the solutions proposed in state of the art can be PWM topologies as in $[1,5]$, multilevel converters, as in [4] or hybrid solutions that employ linear regulators [6].

The main drawbacks of using a PWM converter for this kind of applications rely on the complexity of the design of the ontput filter and the control [2]. The output filter shonld have an adequate size in order to accomplish certain requirements of current and voltage ripples but should also allow very fast changes in the ontput voltage level. These requirements can be considered to be contradictory. A converter operating with very high operating frequency ( $\mathrm{MHz}$ range) is necessary, along with a very fast control loop. These increase the complexity of the PWM power snpply.

The PWM multiphase topology proposed in this paper, could avoid some of these drawbacks. The proposed topology is a multiphase converler with magnetic coupling among the phases. Its operating principle (presented in [3]) will be reviewed in next section. It is important to point ont that in this converter the phases are coupled with transformers instead of coupled inductors ([7]). This implies that the storage of energy in the converter is minimized and that the energy transfer between the inpnt and the ontpnt is very fast, so the output voltage can be changed with a very high slew rate. When this concept is applied, the converter can accomplish an adeqnate filtering of the ontput along with a high bandwidth while working in open loop. 


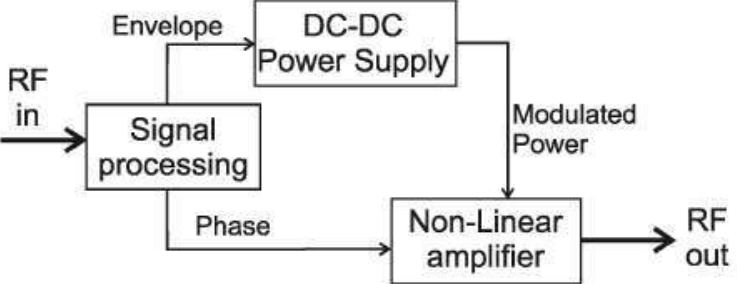

a)

b)

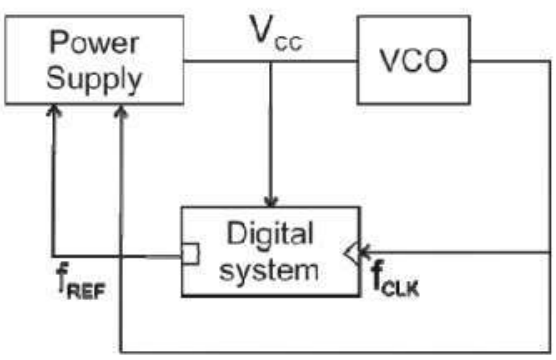

Figure 1. a) Simplified scheme of an RF system: Power Supply for the linear amplifier must supply a modulated output voltage in order to maximize the overall efficiency of the system. b) System architecture for DVS implementation [1]. In both systems, adjusting the power supply output can lead to a more efficient processing of the energy.

Under these conditions of minimum energy storage, the dynamic response is decoupled from the operating frequency and some of the limitations of a PWM converter when used for saving power in RF systems can be avoided.

The paper is organized as follows. The application of the proposed concept as a fast output voltage adjusting power supply is presented in Section II, along with the operating principle of the topology. Section III presents an example of an implementation of the proposed topology; measurements of the output voltage, following a $500 \mathrm{kHz}$ sinusoidal waveform reference are also presented. In Section IV the conclusion of the paper is presented.

\section{POWER ANALOG TO DIGITAL CONVERTER: OPERATING PRINCIPLE}

As mentioned above, when using PWM converters for voltage modulation techniques, such as EER, one of the main drawbacks is the complex design of the feedback and the output filter $[2,8]$. This design is a direct tradeoff between the achievable bandwidth of the converter and the filtering of the output ripple [1].

It is well known that tight filtering needs can be easily met with a high value of inductor that will allow the reduction of current ripple. However, the energy stored in this inductor will limit the energy transfer when a load change or voltage step is demanded to the converter. It is also well known that the frequency rising allows the use of small inductor and capacitor values, while accomplishing tight filtering and enough bandwidth. The main drawback when rising operating frequency of power converters is that switching losses also increase, degrading the efficiency of the converter and hence the overall efficiency of the system where the power supply is placed. In order to find a better trade-off among dynamic response, filtering and efficiency, multiphase converters were proposed in state of the art [9]. Thanks to current ripple cancellation in multiphase converters, smaller inductance values can be used, while still accomplishing filtering needs.

The proposed topology can be described as a multi-phase converter with magnetic coupling among its phases. In it, the phases of the converter are coupled using discrete transformers rather than coupled inductors. A schematic of the topology is shown in Figure 2.b. In this figure, the ideal representation of the transformers that couple the phases is presented; the set of these transformers can be treated as a unique magnetic structure. An adequate control of the energy flowing from the input should ensure that the sum of the input voltages to the magnetic structure $\left(\mathrm{v}_{1}, \mathrm{v}_{2}, \mathrm{v}_{3}\right.$ and $\mathrm{v}_{4}$ in Figure 2b) should be held constant for every instant of time.

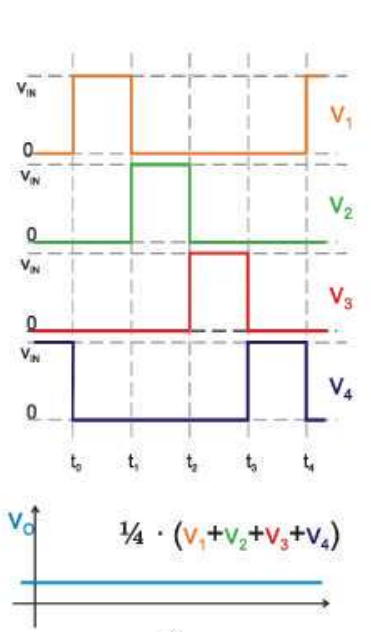

a)

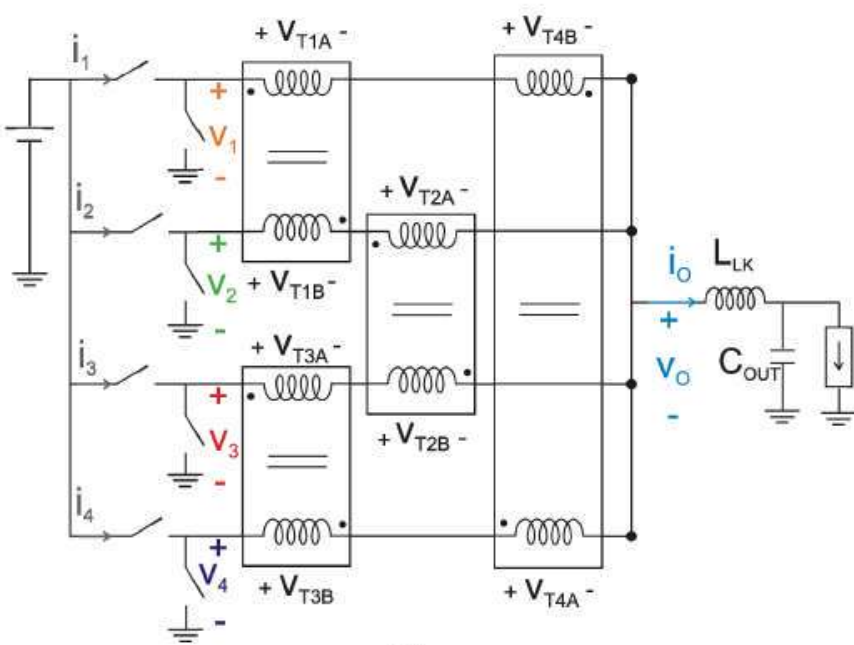

b)

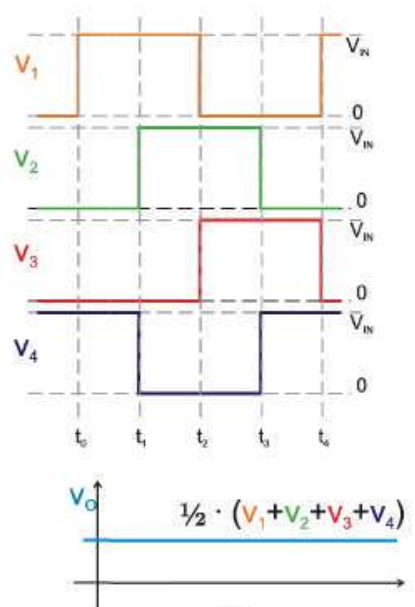

c)

Figure 2. a) Operating waveforms for proposed topology when $\mathrm{d}=25 \%$ the sum of this waveforms results in a constant voltage for every instant of time. b) Schematic diagram of proposed topology with four phases. c) operating voltage waveforms for $d=50 \%$ 


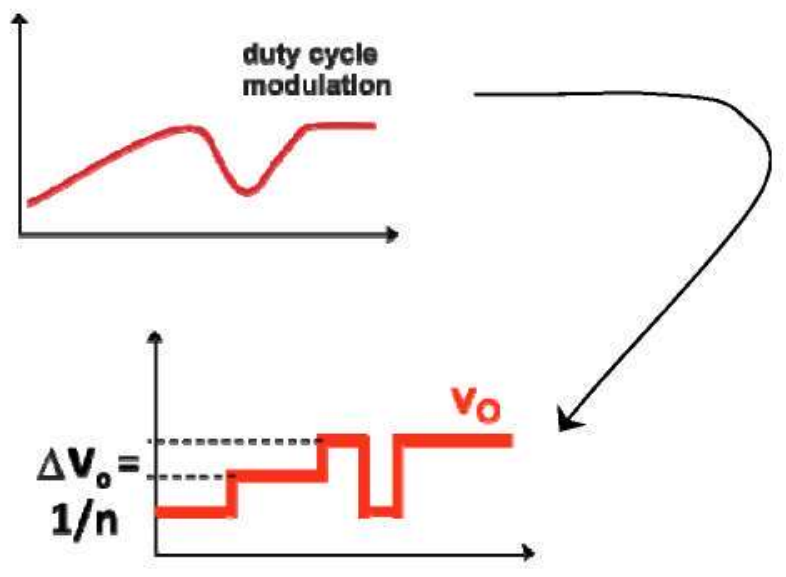

Figure 3. The output voltage of the proposed topology can be changed only within discrete values, this enables the use of the proposed topology as a power $\mathrm{ADC}$ where the resolution is given by $1 / \mathrm{n}$ (where $\mathrm{n}$ is the number of phases.)

The sum of the voltages at the input will result in a constant voltage at the output of the magnetic structure $\left(\mathrm{v}_{\mathrm{C}}\right)$.

The general principle of operation can be described by the following equations:

$$
\begin{gathered}
v_{C}=\frac{1}{n} \cdot \sum v_{i}(t) \\
i_{i}=\frac{t_{O}}{n}
\end{gathered}
$$

where $n$ is the total number of phases. The validation of this concept is presented in [3] where it is applied to a two-phase converter. In order to use transformers to couple the phases of the converter, the energy flow to the magnetic structure should be kept constant for every instant of time. This means that the proposed topology can operate only at the duty cycles where the sum of the input voltages to the magnetic structure is constant. These operating points can be referred as operation nodes. The number of available operation nodes is related to the number of the phases in the converter; the available operation nodes are given by:

$$
\mathrm{d}=\mathrm{k} / \mathrm{n}
$$

where $k$ is an integer number whose values range between $l$ and $n-1 ; k$ represents the number of phases which are simultaneously transferring energy from the input.

This topology operates in open loop; hence, the changes in the output voltage are achieved by changing the value of $k$ between 1 and $n-1$. The minimum change between consecutive operation nodes will be set by the number of phases. This value is given by $1 / n$. Figure 3 illustrates this concept. If duty cycle of the converter is modulated with an analog signal, the output of the power supply will follow it within discrete steps. Because of this feature, it can be said that this topology can be considered as a Power Analog to Digital Converter where the resolution depends on the number of phases.
The application of this control strategy to a four-phase topology is illustrated in Figure 2. Since it is a four-phase topology, there are three operating nodes with duty cycles: $25 \%, 50 \%$ and $75 \%$. Figure $2 \mathrm{a}$ shows the case where duty cycle is $25 \%$. The operation of the topology over one switching cycle has been divided into four instants of time:

- During $t_{0}-t_{1}$, phase 1 is connected to $V_{I N}$ and transfers energy from the input to the magnetic structure, while the other phases are connected to ground. At $t_{1}$, phase 1 changes from $V_{I N}$ to ground, and at the same time, phase 2 changes from ground to $V_{I N}$, since the energy flow must remain constant for every instant of time.

- During $t_{1}-t_{2}$, phase 2 transfers energy from $V_{\mathbb{I N}}$ to the magnetic structure, making $V_{2}$ equal to $V_{I N}$.

The same process is repeated for phases 2 and 3 during $t_{2}$ $t_{3}$ and $t_{3}-t_{4}$. Table I summarizes the values of the input voltages to the magnetic structure for every instant of time, and Table II shows the value of the voltages of each transformer for the same instants of time.

In this operation point and for every time interval, there is only one phase transferring energy to the magnetic structure at every instant of time, hence in equation (1), $\mathrm{k}=1$ and the resulting duty cycle is $1 / 4(25 \%)$. The sum of the input voltages $\left(v_{1}+v_{2}+v_{3}+v_{4}\right)$ results in a constant input voltage to the magnetic structure for every instant of time. If the input voltage to the magnetic structure is constant, $v_{O}$ will be also constant and equal to:

$$
(\mathrm{v} 1+\mathrm{v} 2+\mathrm{v} 3+\mathrm{v} 4) / \mathrm{n}
$$

In this case and for a duty cycle of $25 \%, v_{\mathrm{O}}=1 / 4 \cdot \mathrm{V}_{\mathrm{IN}}$.

The next operation node corresponds to $\mathrm{d}=50 \%$. The operating principle is the same than that for $d=25 \%$. Except that, instead of one phase transferring energy, there are two phases simultaneously connected to $\mathrm{V}_{\mathrm{IN}}$ and transferring energy to the magnetic structure in order to keep the sum of the input voltages constant for every instant of time. If two cells are connected to $V_{\mathbb{I N}}$ the input voltage to the magnetic structure is the double than that of the former case:

$$
2 \cdot(v 1+v 2+v 3+v 4) / n
$$

and since duty cycle equals $50 \%, \mathrm{v}_{\mathrm{O}}=1 / 2 \cdot \mathrm{V}_{\mathrm{IN}}$.

When duty cycle is equal to $75 \%$ there would be three phases transferring energy to the magnetic structure for every instant of time, and $v_{O}=3 / 4 \cdot V_{I N}$. For these three duty cycles, $\mathrm{v}_{\mathrm{O}}$ remains constant for every instant of time before the output filter of the converter and it is ideally not necessary to design the size of its components in order to filter the output ripple. In fact, the output inductor is comprised only by the equivalent leakage inductance of the set of transformers (represented in Figure 2 by $\mathrm{L}_{\mathrm{LK}}$ ). The value of this inductance can be minimized (to tenths of $\mathrm{nH}$ ) if the adequate interleaving technique is chosen when placing the windings in the transformer. 
TABLE I. PHASE VOLTAGES FOR EVERY INSTANT OF TIME

\begin{tabular}{|l|c|c|c|c|}
\hline \multirow{2}{*}{$\begin{array}{c}\text { Time } \\
\text { Instant }\end{array}$} & \multicolumn{4}{|c|}{ Input Voltages to the magnetic structure } \\
\cline { 2 - 5 } & $\boldsymbol{v} 1$ & $\boldsymbol{v} 2$ & $\boldsymbol{v} 3$ & $\boldsymbol{1} 4$ \\
\hline $\mathrm{t} 0-\mathrm{t} 1$ & $\mathrm{~V}_{\mathrm{IN}}$ & 0 & 0 & 0 \\
\hline $\mathrm{t} 1-\mathrm{t} 2$ & 0 & $\mathrm{~V}_{\mathrm{IN}}$ & 0 & 0 \\
\hline $\mathrm{t} 2-\mathrm{t} 3$ & 0 & 0 & $\mathrm{~V}_{\mathrm{IN}}$ & 0 \\
\hline $\mathrm{t} 3-\mathrm{t} 4$ & 0 & 0 & 0 & $\mathrm{~V}_{\mathrm{IN}}$ \\
\hline
\end{tabular}

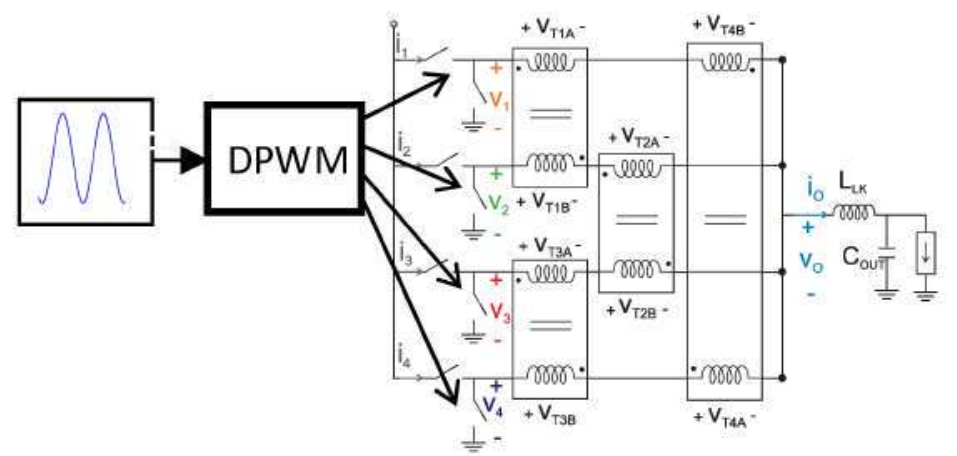

TABLE II.TRANSFORMER VOLTAGES FOR EVERY INSTANT OF TIME, CORRESPONDING TO $\mathrm{D}=25 \%$

\begin{tabular}{|l|c|c|c|c|}
\hline \multirow{2}{*}{$\begin{array}{c}\text { Time } \\
\text { Instant }\end{array}$} & \multicolumn{4}{|c|}{ Transformer Voltages } \\
\cline { 2 - 5 } & $V T 1$ & $V T 2$ & $V T 3$ & $V T 4$ \\
\hline $\mathrm{t} 0-\mathrm{t} \mathrm{l}$ & $3 / 8 \cdot \mathrm{V}_{\mathrm{IN}}$ & $1 / 8 \cdot \mathrm{V}_{\mathrm{IN}}$ & $-1 / 8 \cdot \mathrm{V}_{\mathrm{IN}}$ & $-3 / 8 \mathrm{~V}_{\mathrm{IN}}$ \\
\hline $\mathrm{t} 1-\mathrm{t} 2$ & $-3 / 8 \mathrm{~V}_{\mathrm{IN}}$ & $3 / 8 \cdot \mathrm{V}_{\mathrm{IN}}$ & $1 / 8 \cdot \mathrm{V}_{\mathrm{IN}}$ & $-1 / 8 \cdot \mathrm{V}_{\mathrm{IN}}$ \\
\hline $\mathrm{t} 2-\mathrm{t} 3$ & $-1 / 8 \cdot \mathrm{V}_{\mathrm{IN}}$ & $-3 / 8 \mathrm{~V}_{\mathrm{IN}}$ & $3 / 8 \cdot \mathrm{V}_{\mathrm{IN}}$ & $1 / 8 \cdot \mathrm{V}_{\mathrm{IN}}$ \\
\hline $\mathrm{t} 3-\mathrm{t} 4$ & $1 / 8 \cdot \mathrm{V}_{\mathrm{IN}}$ & $-1 / 8 \cdot \mathrm{V}_{\mathrm{IN}}$ & $-3 / 8 \mathrm{~V}_{\mathrm{IN}}$ & $3 / 8 \cdot \mathrm{V}_{\mathbb{I N}}$ \\
\hline
\end{tabular}

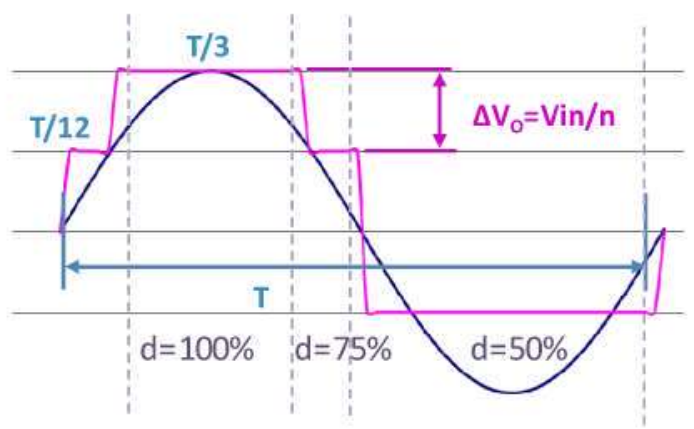

Figure 4. A sinusoidal reference is used to modulate the duty cycle applied to the converter, the resulting output voltage follows the sinusoidal reference within discrete steps.

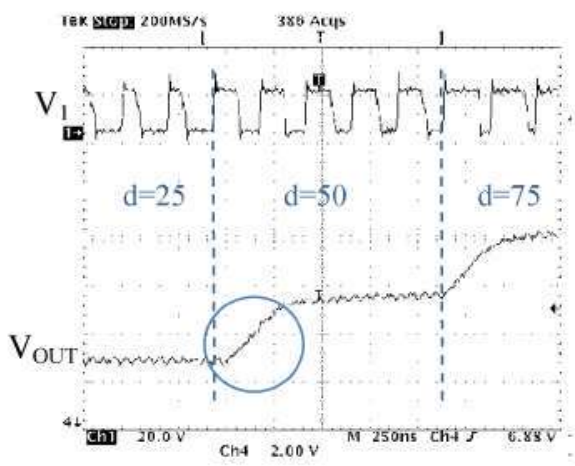

Figure 5. dv/dt of approx. $8 \mathrm{~V} / \mu \mathrm{s}$ is achieved under no load condition, $\mathrm{f}_{\mathrm{sw}}=4 \mathrm{MHz}$ and an output voltage change from $3 \mathrm{~V}$ to $6 \mathrm{~V}$ and an output capacitor of $1 \mu \mathrm{F}$. $V_{1}$ represents the voltage of phase 1 as seen by the input of the magnetic structure. $V_{\text {OUT }}$ represents the output voltage of the converter

\section{APPLICATION EXAMPLE: ENVELOPE POWER SUPPLY FOR RF AMPLIFIER}

In order to validate this concept in the area of modulated power supplies for RF applications, the RF system presented in [4], where the EER technique is implemented, was selected in order to evaluate the application of the proposed topology as a part of the aforementioned RF system.

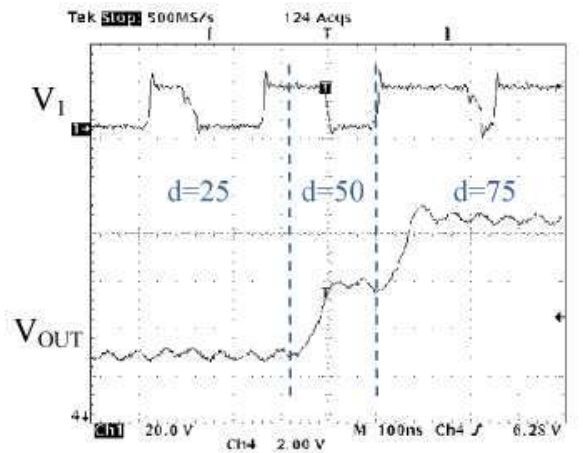

Figure 6. Output voltage waveform of the converter for $\mathrm{f}_{\mathrm{SW}}=2 \mathrm{MHz}$ and a capacitor of $47 \mathrm{nF}$. The frequency of the sinusoidal waveform that modulates the duty cycle is $170 \mathrm{KHz}_{1}$ represents the voltage of phase 1 as seen by the input of the magnetic structure. $V_{\text {OuT }}$ represents the output voltage of the converter

The configuration of the envelope amplifier of the system presented in [4] is comprised of three stages: a multiple output dc-dc converter that provides stable dc voltages; a multilevel converter, which is used to adjust different voltage levels and a linear regulator in series with the multilevel converter. In the same way, a system for an envelope amplifier could be built with the proposed topology plus the linear regulator. 


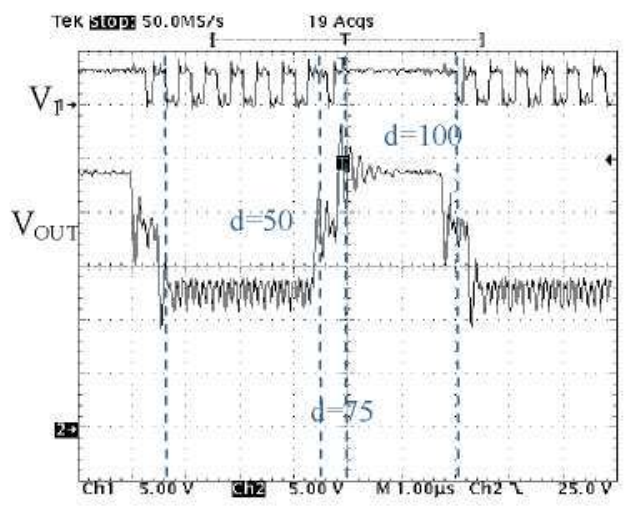

Figure 7. Output voltage waveform of the converter for $\mathrm{f}_{\mathrm{Sw}}=4 \mathrm{MHz}$, sinusoidal waveform is $170 \mathrm{kHz} . V_{1}$ represents the voltage of phase 1 as seen by the input of the magnetic structure. VOur represents the output voltage of the converter

Although the filter of the converter can be designed independently from the filtering needs, and the dynamic response of the system is decoupled from switching frequency, the maximum frequency of the sinusoidal reference that modulates the duty cycle is still limited by the switching frequency of the converter. So, at the operating frequency of 2 $\mathrm{MHz}$, the maximum frequency of the sine wave that can be tracked is around $200 \mathrm{kHz}$.

A block diagram of the configuration of the power supply as a part of an RF system power supply is shown in Figure 4. It can be seen that the system operates in open loop and that a sinusoidal reference is used to modulate the duty cycle. The resulting output voltage modulation is also shown in this figure.

Since this topology operates in open loop, the slew rate of the output voltage can be approximated as the response of a second order system where its main components are the output inductor and capacitor. The value of the output inductor is fixed by the equivalent leakage inductance of the transformers set, and then the value of the output capacitor will define if the system response to a duty cycle change is fast and underdamped or slower and over-damped. Figure 5 and Figure 6 show two slew rates measured at $\mathrm{f}_{\mathrm{SW}}=4 \mathrm{MHz}$, these slew rates correspond to different output capacitor values. It can be seen that the fastest slew rate is achieved with a $47 \mathrm{nF}$ MLC Capacitor. It is important to point out that in this application, current load steps are not a concern.

The configuration of the system is as follows: $V_{\mathbb{N}}=24 \mathrm{~V}$, $\mathrm{f}_{\mathrm{SW}}=2 \mathrm{MHz}, \mathrm{P}_{\text {OUTMAX }}=30 \mathrm{~W}$. The duty cycle is modulated by a sinusoidal waveform of $170 \mathrm{kHz}$; the resulting sequence of duty cycles is as follows: $50 \%-75 \%-100 \%-75 \%$ and the measured efficiency at $2 \mathrm{MHz}$ is around $87 \%$. The configuration of the magnetic structure corresponds to that shown in Figure $3 \mathrm{~b}$. The results of modulating the duty cycle with a sinusoidal waveform are shown in Figure 7 and Figure 8 , the resulting output waveform is shown at $\mathrm{f}_{\mathrm{Sw}}=2 \mathrm{MHz}$ and $\mathrm{f}_{\mathrm{SW}}=4 \mathrm{MHz}$, respectively. The configuration of the final system includes a linear regulator. This linear regulator filters

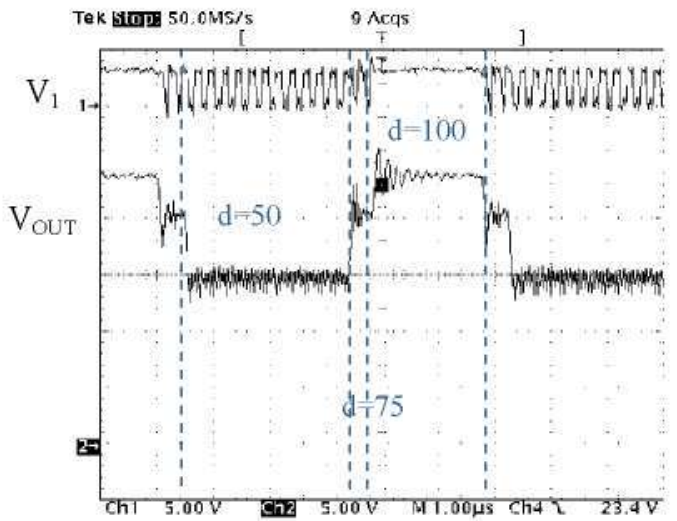

Figure 8. $\mathrm{dv} / \mathrm{dt}$ of approx. $16 \mathrm{~V} / \mu \mathrm{s}$ is achieved under no load condition, $\mathrm{fSW}=4 \mathrm{MHz}$ and an output voltage change from $3 \mathrm{~V}$ to $6 \mathrm{~V}$ an output capacitor of $33 \mathrm{uF}$. $V_{1}$ represents the voltage of phase 1 as seen by the input of the magnetic structure. Vour represents the output voltage of the converter

the noise that comes from the converter and provides fine adjustment of the output voltage of the envelope amplifier.

\section{CONCLUSIONS}

In this paper, a PWM multiphase topology based on transformer-coupling was presented along with an application for voltage modulation. In this multiphase topology, the coupling between phases is done by using transformers instead of coupling inductors. Since transformers do not store energy, the transfer of the energy between the input and the output is done very fast, which allows a very fast change of the output voltage.

This topology can be useful when integrated in RF systems where power modulation techniques, such as envelope elimination and restoration (EER) are employed in order to increase the system efficiency. A demonstrator, where the duty cycle is modulated with a $170 \mathrm{kHz}$ sinusoidal waveform has been presented, showing an efficiency of $87 \%$ and a correct tracking of the modulating waveform. The output power for this application is $30 \mathrm{~W}$.

\section{ACKNOWLEDGMENT}

The authors would like to thank Narciso Ferreros Sánchez whose participation in the development and measurement of the aforementioned prototype were very valuable to this work.

\section{REFERENCES}

[1] A.Soto, P.Alou, J.A.Cobos and J.Uceda, "The future DC-DC Converter as an Enabler of Low Energy Consumption Systems with Dynamic Voltage Scaling", in IECON 02 Industrial Electronics Society, IEEE 2002 28th Annual Conference.

[2] L.Marco, E.Alarcón and D.Maksimovic, "Effects of switching power converter non-idealities in Envelope Elimination and Restoration 
technique", in Circuits and Systens, 2006. ISCAS 2006. Proceedings. 2006 IEEE Intenational Symposium on

[3] M.C.Gonzalez, L.Lagua, P.Alou, O.Garcia, J.A.Cobos and H.Visairo, "New control strategy for energy conversion based on coupled inagnetic structures". Power Elestronics Specialists Conference, 2008. PESC 2008

[4] M.Vasic, O.Garcia, J.A.Oliver, P.Alou, D.Diaz and J.A.Cobos, "Multilevel Power Supply for High Efficiency RF Amplifiers", Applied Power Electronics Conference and Exposition, 2009. APEC 2009. Twenty-Fourth Annual IEEE pp 1233 - 1238

[5] Mikkel C. W. Høyerby and Michael A. E. Andersen: "Ultrafast Tracking Power Supply With Fourh-Order Output Filter and FixedFrequency Hysteretic Control", IEEE Transactions On Power Electronies, Vol. 23, No. 5, September 2008

[6] V. Yousefzadeh. E. Alarcon. D. Maksimovic,; "Effìciency optitnization in linear assisted switching power converers for envelope tracking in RF power amplifiers". IEEE International Syinposium on Circuits and Systens, ISCAS 2005, pages:1302-1305 Vol. 2

[7] Pit-Leong Wong, Peng Xiv, P.Yang, and F.C.Lee, "Perfornance inprovements of interleaving VRMs with coupling indktors". IEEE Transactions on Power Electronics. Jul 2001

[8] Mikkel C. W. Hoyerby, Michael A. E. Andersen, "Optimized Envelope Tracking Power Supply for Tetra2 Base Station RF Power Anplifier". Applied Power Electronics Conference and Exposition. 2008. APEC 2008

[9] Oliver J.A., Zumel P., Garcia O., Cobos J.A. and Uceda J. "Passive component analysis in imterleaved buck converters". Applied PowerElectronics Conference and Exposition, 2004. APEC '04. Nineteenth Annual IEEE 